\title{
ON INFLUENCE AND COMPROMISE IN TWO-TIER VOTING SYSTEMS
}

\author{
GEOFFREY R. GRIMMETT
}

\begin{abstract}
We examine two aspects of the mathematical basis for two-tier voting systems, such as that of the Council of the European Union. These aspects concern the use of square-root weights and the choice of quota.

Square-root weights originate in the Penrose square-root system, which assumes that votes are cast independently and uniformly at random, and is based around the concept of equality of influence of the voters across the Union. There are (at least) two distinct definitions of influence in current use in probability theory, namely, absolute and conditional influence. These are in agreement when the underlying random variables are independent, but not generally. We review their possible implications for two-tier voting systems, especially in the context of the so-called collective bias model. We show that the two square-root laws invoked by Penrose are unified through the use of conditional influence.

In an elaboration of the square-root system, Słomczyński and Życzkowski have proposed an exact value for the quota $q=q^{*}$ to be achieved in a successful vote of a two-tier system, and they have presented numerical and theoretical evidence in its support. We indicate some numerical and mathematical issues arising in the use of a Gaussian (or normal) approximation in this context, and we propose that other values of $q$ may be as good if not better than $q^{*}$. We discuss certain aspects of the relationship between theoreticians and politicians in the design of a two-tier voting system, and we reach the conclusion that the choice of quota in the square-root system is an issue for politicians informed by theory.
\end{abstract}

\section{INTRODUCTION AND BACKGROUND}

1.1. Preamble. Mathematics is fundamental to the design and analysis of voting systems (see, for example, the books $[3,10,16,36,43]$ ). Mathematical models for human behaviour frequently involve probability, and they invariably rely upon assumptions whose validity is ripe for debate. As a general rule, the greater are the assumptions, the more precise are the conclusions. A balance needs be struck between tractability and applicability: excessive assumptions tend to undermine practical

Date: 21 March 2018, revised 14 May 2019.

2010 Mathematics Subject Classification. 60F05, 91B12.

Key words and phrases. Two-tier voting system, European Union, Council, influence, power, pivotality, collective bias, Jagiellonian Compromise, Berry-Esseen bound. 
relevance, whereas "nothing will come of nothing" [Shakespeare]. Assumption-based conclusions must be exposed to a robustness analysis: to what degree are they robust when the underlying assumptions are perturbed?

These issues are illustrated here in a study of the so-called Jagiellonian Compromise (JagCom) of Słomczyński and Życzkowski [49, 50, 51, 55]. The JagCom is a proposal for a two-tier voting system such as that of the Council of the European Union (see Section 1.3 for further details). It is based on (i) square-root weights coupled with (ii) a certain formula for the quota. While the current work was born out of an interest in learning about the JagCom, it has developed into (i) a broader study of the notion of power (or influence) for general probability distributions, combined with (ii) a critical analysis of the arguments leading to the given quota. The conclusions of this article illuminate the balance between theory and practical relevance.

The political context of this paper is as follows. The debate rolls on concerning the allocation of seats in the European Parliament (EP) between the Member States of the EU (see, for example, $[12,44]$ ). It has been argued by members of the Constitutional Affairs Committee (AFCO) of the EP that strategic reform of the somewhat ad hoc method of allocation of parliamentary seats should be considered only in parallel to a review of the two-tier voting system of the EU Council. The JagCom is a leading theoretical contender for implementation in the Council. It has been supported in two open letters to EU governments, [8, 9], signed by significant numbers of prominent theoreticians, and it has been the subject of a volume of positive publicity including [30, 38, 45, 52, 56]. The discussion in Brussels is likely to intensify in the months and years to come, and this is a propitious moment to re-examine the JagCom in some detail.

There are two principal parts to this paper, as outlined in the following two subsections. The first concerns the definition of the 'power' of an individual (as introduced by Penrose) for general probability distributions. This is connected to the choice of weights in a two-tier voting system such as the JagCom. The second is a discussion of the choice of quota in the JagCom.

1.2. Power and influence. Lionel Penrose's 1946 paper [41] is a fundamental work in the mathematical theory of voting, and it has received a great deal of attention. Penrose found it convenient to assume that members of a population choose their votes independently at random, and are equally likely to choose either of the two possible outcomes. These assumptions of independence and unbiasedness lead to a mathematically sophisticated theory based around the classical study of the sums of so-called independent and identically distributed ('iid'), symmetric random variables (see [42], or the less sophisticated account [24, Sect. 5.10]). That said, independence and unbiasedness may, in practice, be far from the truth in specific cases. 
The square-root voting system of Penrose [41] is prominent in discussion of two-tier voting systems in general, and in specific of that of the Council of the European Union (see, for example, $[27,55]$ ). The challenge confronted by Penrose is to devise a system for pooling the views of a number of Member States with varying population sizes. What weight $w_{j}$ should be assigned to the opinion of State $j$, having a population of size $N_{j}$ ? The Penrose system amounts to the proposal $w_{j} \propto \sqrt{N_{j}}$. The essence of Penrose's argument is the observation that the number $H$ of heads shown in $N_{j}$ independent, unbiased coin tosses satisfies

$$
\mathbb{E}\left|H-\frac{1}{2} N_{j}\right| \sim \sqrt{2 N_{j} / \pi}, \quad \text { for large } N_{j} .
$$

(Here and later, $\mathbb{E}$ denotes expectation, and $\mathbb{P}$ denotes probability.) We shall refer to (1.1) as Penrose's second square-root law. The reader is referred back to [41] for the deduction of square-root weights from (1.1), although s/he may prefer to read Kirsch's least-squares argument as presented in Section 3.2. (Although (1.1), and the subsequent (1.2), are asymptotic relations, sharp bounds may be obtained by elementary methods.)

Remark 1.1. Penrose defines the 'edge' as $\left|N_{F}-N_{A}\right|$, where $N_{F}$ (respectively, $N_{A}$ ) is the number of votes in favour (respectively, against) the motion. It is immediate that $\left|N_{F}-N_{A}\right|=\left|H-\left(N_{j}-H\right)\right|=\left|2 H-N_{j}\right|$, so that the mean edge equals $2 \mathbb{E}\left|H-\frac{1}{2} N_{j}\right|$.

Penrose [41] discussed also the concept of the 'power' (termed 'influence' in the current work, after [6, 47]) of an individual voter within a given election or vote. He noted that, in a vote within a State containing $N_{j}$ individuals whose votes are independent and unbiased, this power, denoted $\alpha_{j}$, has order

$$
\alpha_{j} \sim \sqrt{2 /\left(\pi N_{j}\right)}, \quad \text { for large } N_{j},
$$

(see also Banzhaf [4]). We shall refer to (1.2) as Penrose's first square-root law.

Only one square-root is sometimes attributed to Penrose. In [41], he stated (1.1) and he proved (1.2), and he did not note their inter-relationship. Some later authors have linked (1.1) and (1.2) by proposing a weight $w_{j}^{\prime}$ for State $j$ such that the product $\alpha_{j} w_{j}^{\prime}$ does not depend on population-size, that is, $w_{j}^{\prime} \propto \sqrt{N_{j}}$, in agreement with (1.1) (see, for example, the discussion at [52, p. $48 \mathrm{ff}$.] and [55, Sect. 1]). This argument appears to assume that: (i) in a population with size $N_{j}$ and individual power $\alpha_{j}$, the collective power is $\alpha_{j} N_{j}$, and (ii) $1 / \alpha_{j}$ has, generically, the same order as $\mathbb{E}\left|H-\frac{1}{2} N_{j}\right|$. The first assumption here is open to discussion, and the second is false for general distributions. Proposition 2.5 and Remark 2.6 explain the true relationship between (1.1) and (1.2) in the context of general probability distributions. 
The square-root laws of this article are those of Penrose [41]. It is not the current purpose to discuss in detail the relationship between voting power and voting weight (see, for example, $[2,16,39]$ ).

In Section 2, we introduce the notions of the absolute and the conditional influences of an individual in an election. The absolute influence is that considered by Penrose and later authors; the conditional influence is sometimes considered more appropriate in situations where individuals' votes are dependent random variables. The two quantities are equal in the independent case, but not generally so.

1.3. The Jagiellonian Compromise, a two-tier voting system. In a method since dubbed the 'Jagiellonian Compromise' (JagCom), Słomczyński and Życzkowski $[49,50,51,55]$ have proposed the following two-tier voting system using square-root weights together with a particular value $q^{*}$ for the quota $q$. Writing $N_{1}, N_{2}, \ldots, N_{s}$ for the populations of the $s$ States of the Union, under the JagCom a motion is passed if and only if

$$
\sum_{j \in J} \sqrt{N_{j}}-\sum_{j \in \bar{J}} \sqrt{N_{j}}>q^{*} W
$$

where $J$ is the set of States voting in favour of the motion, $\bar{J}$ is the set voting against, and

$$
q^{*}:=\frac{\sqrt{N}}{W}, \quad W=\sum_{j=1}^{s} \sqrt{N_{j}}, \quad N=\sum_{j=1}^{s} N_{j} .
$$

The value $q=q^{*}$ given in (1.3) is supported by a heuristic argument based on approximation by a Gaussian distribution. Although no rigorous justification of this approximation is yet available (see Appendix $\mathrm{C}$ of the current work), its conclusions gain some support using exact numerical methods (see Section 4.2). Note that the $q^{*}$ of (1.3) is not quite the quota of [51], but rather that of [26].

Salient features of two-tier voting systems are summarised in Section 3, with special attention to the work of Kirsch [26, 27] and Słomczyński and Życzkowski [49, 50, 51, 55]. This is followed by a discussion in Section 4 and Appendix $\mathrm{C}$ of the influences of the weighted States within the Council, and of the use and potential misuse of the Gaussian approximation in estimating certain related probabilities. The closing Section 5 contains some reflections on the JagCom, and in particular the following conclusions.

1. The JagCom is derived via a set of principles that can be stated unambiguously and analysed rigorously, and the resulting system is robust with respect to population changes. Nevertheless, these principles are arguably fragile and unrealistic, and insufficiently sensitive to political realities. 
2. Despite fragility in the assumptions about voting patterns made by the JagCom, we offer no superior alternative here. The problem of allocating weights is more than just a mathematical puzzle, but demands a more extensive political vision.

3. The justification for the proposed JagCom quota $q^{*}$ is empirical and numerical rather than rigorous. These numerics provide only equivocal guidance which is satisfied by a range of values of the quota, including the simpler value $q=0$. Indeed, $q=0$ is the value for which individual powers are maximized. Given the very modest differences in performance between such values of the quota, the final choice is best determined through political input.

1.4. Resumé. Certain assumptions appear to be necessary for the above analyses, and these are examined in the current article. There are four areas that receive special attention, namely:

(a) the underlying model in which individuals vote according to an unbiased coin toss, independently of other voters [Section 2.3],

(b) an alternative interpretation of the concept of 'voting power' or 'influence' [Section 2.2],

(c) the assumptions of mathematical smoothness under which the Gaussian approximation is suitable for finite populations [Appendix C],

(d) some implications of exact computations of voting powers in two-tier systems [Section 4.2].

Numerous earlier authors have of course considered some of these issues, namely (a), (c), and (d), and we mention [27, 31, 35, 37, 46, 51].

Remark 1.2 (on the literature). There is an extensive existing literature on the matters considered in this article, and the author has attempted to include appropriate references. Apologies are offered to those authors whose work is not listed explicitly.

\section{Absolute AND CONDitional INFLUENCE}

2.1. The history and context of influence. The concept of 'influence' is central in the probability theory of disordered systems. Consider a system that comprises $m$ sub-systems. These could be, for example, individual voters in an election, nodes in a disordered medium (as in the percolation model), or particles in a model for the ferromagnet (such as the Ising/Potts models). In studying the behaviour of the collective system, it is often key to understand the effect of a variation within a given sub-system. That is, what is the probability that a change in a given sub-system has a substantial effect on the collective system?

The quantification of influence is long recognised as being central to the understanding of complex random systems. For example, influence in voting systems was 
studied by Ben-Or and Linial [6] in work that played an important role in stimulating a systematic theory of influence and sharp threshold with many applications in random systems (see [25] for a review). In percolation theory, the influence of a node is the probability that the node is pivotal for a given global event (see (2.3) for the definition of pivotality). Estimates for influence are key to most of the principal results for percolation (see [21], for example). In these two areas above, the sub-systems are generally taken to be independent random variables. This is, however, not so for a number of important processes of statistical mechanics including the Ising and Potts models, in which the sub-systems are dependent but usually positively correlated. For such systems, 'influence' requires a new definition, and this is provided in $[19,20]$ in the context of the Ising and random-cluster models (see [22]).

The origins of influence are rather older than the above work, and go back at least to the work of Penrose [41] in 1946 and possibly the reliability literature surveyed by Barlow and Proschan [5] in 1965. The two definitions of influence, referred to above, are presented next in the context of a voting system (we shall use the standard terminology of probability theory and the theory of interacting systems).

2.2. Definitions of absolute and conditional influences. There is a population $P$ containing $m$ individuals, and a vote is taken between two possible outcomes, labelled +1 and -1 . Each individual votes either +1 or -1 . We write $X(i)$ for the vote of person $i$, and we assume the $X(i)$ are random variables. The votevector $X=(X(1), X(2), \ldots, X(m))$ takes values in the so-called 'configuration space' $\Sigma=\{-1,1\}^{m}$. There exists a predetermined subset $A \subseteq \Sigma$, and the vote is declared to pass if and only if $X \in A$. It is normal to consider sets $A$ which are increasing in that

$$
\sigma \in A, \sigma \leq \sigma^{\prime} \Rightarrow \sigma^{\prime} \in A .
$$

The inequality $\sigma \leq \sigma^{\prime}$ refers to the natural partial order on $\Sigma$ given by

$$
\sigma \leq \sigma^{\prime} \text { if and only if } \sigma(i) \leq \sigma^{\prime}(i) \text { for all } i \in P \text {. }
$$

For concreteness, we assume henceforth that $A$ is an increasing subset of $\Sigma$ (that is, an 'increasing event').

For $i \in P$ and a configuration $\sigma=(\sigma(1), \sigma(2), \ldots, \sigma(m)) \in \Sigma$, we define the vectors $\sigma^{i}, \sigma_{i}$ by

$$
\sigma^{i}(j)=\left\{\begin{array}{ll}
1 & \text { if } j=i, \\
\sigma(j) & \text { otherwise, }
\end{array} \quad \sigma_{i}(j)= \begin{cases}-1 & \text { if } j=i, \\
\sigma(j) & \text { otherwise }\end{cases}\right.
$$

That is, $\sigma^{i}$ (respectively, $\sigma_{i}$ ) agrees with $\sigma$ except possibly at $i$, with $i$ 's vote set to 1 (respectively, -1 ). Individual $i$ is called pivotal if the outcome of the vote changes 
when s/he changes opinion (the words decisive and critical are sometimes used in the voting literature). More formally, $i$ is called pivotal for the configuration $\sigma$ if

$$
\sigma_{i} \notin A, \quad \sigma^{i} \in A .
$$

This holds since $A$ is assumed increasing.

In all situations considered in this paper, the individual votes $X(i)$ are assumed to be identically distributed and symmetric in that

$$
\mathbb{P}(X(i)=1)=\mathbb{P}(X(i)=-1)=\frac{1}{2},
$$

where $\mathbb{P}$ denotes the probability measure that governs the vote-vector $X$. Assumptions of independence will be introduced where appropriate.

Definition 2.1. We say that the vector $X$ is symmetric if

(i) $X$ and $-X$ have the same distributions, and

(ii) for all $i \neq j$ there exists a permutation $\pi$ of $\{1,2, \ldots, m\}$ with $\pi_{i}=j$ such that $X$ and $\pi X$ have the same distribution, where $\pi X$ denotes the permuted vector $\left(X_{\pi_{1}}, X_{\pi_{2}}, \ldots, X_{\pi_{m}}\right)$.

Condition (ii) above is weaker than requiring that $X$ be exchangeable, and an example is included in Appendix A.

Definition 2.2. Let $A$ be an increasing event.

(a) The absolute influence of voter $i$ is

$$
\begin{aligned}
\alpha(i) & :=\mathbb{P}\left(X^{i} \in A\right)-\mathbb{P}\left(X_{i} \in A\right) \\
& =\mathbb{P}\left(X_{i} \notin A, X^{i} \in A\right) .
\end{aligned}
$$

(b) The conditional influence of voter $i$ is

$$
\kappa(i):=\mathbb{P}(A \mid X(i)=1)-\mathbb{P}(A \mid X(i)=-1) .
$$

The so-called 'power index', or the Banzhaf (or Banzhaf-Penrose) power index in the impartial culture context, is the term used by many authors for the absolute influence given above.

When $\mathbb{P}$ is a product measure (that is, the $X(i)$ are independent), it may be seen that $\alpha(i)=\kappa(i)$, and the common value $\alpha$ is termed simply influence by Russo [47] and Ben-Or and Linial [6] (and power by Penrose [41]). Equality does not generally hold when $\mathbb{P}$ is not a product measure. The above concept of 'conditional influence' was identified in [19], where it was shown to be the correct adaptation of absolute influence in proofs of sharp-threshold theorems for certain families of dependent measures arising in stochastic geometry and statistical physics. It has featured recently in work [32] on so-called prediction values within probabilistic games. 
Remark 2.3 (Success probability). The success probability $\eta(i)$ of voter $i$ is the probability that $i$ votes on the winning side, that is,

$$
\eta(i):=\mathbb{P}(A \cap\{X(i)=1\})+\mathbb{P}(\bar{A} \cap\{X(i)=-1\}),
$$

where $\bar{A}$ is the complement of $A$. See, for example, [11,36]. If the $X(i)$ satisfy $(2.4)$, the conditional influence is related to the success probability by the relation

$$
\eta(i)=\frac{1}{2}(1+\kappa(i))
$$

see [16, Thm 3.2.16] and [41, Sect. 2(a)]. This relation is, in fact, the key step in the proof of the forthcoming Proposition 2.5. The success probabilities feature in the recent work of Kirsch [28].

2.3. Examples of influences. There follow three examples of calculations that illustrate the differences between absolute and conditional influence. For convenience, we assume $m=2 r+1$ is an odd number, and take $A$ to be the majority event, that is,

$$
A=\left\{\sigma: \sum_{i} \sigma(i)>0\right\}
$$

It is clear that $A$ is an increasing set. We shall take the $X(i)$ to be Bernoulli random variables with a shared parameter $u$ which may itself be random. Thus, the $X(i)$ are not generally independent.

Let $U$ be a random variable taking values in the interval $(0,1)$. Conditional on the event $U=u$, the $X(i)$ are defined to be independent random variables with

$$
X(i)= \begin{cases}1 & \text { with probability } u, \\ -1 & \text { with probability } 1-u\end{cases}
$$

If $U$ has a symmetric distribution (in that $U$ and $1-U$ are equally distributed), then the ensuing vote-vector $X$ is symmetric (and, indeed, exchangeable), and this is called the 'collective bias' model by Kirsch [26, 27] (see also [29]). Here are three examples of collective bias in which the absolute and conditional influences vary greatly.

1. Independent voting. Let $\mathbb{P}\left(U=\frac{1}{2}\right)=1$. The $X(i)$ are independent, unbiased Bernoulli variables, and

$$
\alpha(i)=\kappa(i)=\left(\begin{array}{c}
2 r \\
r
\end{array}\right)\left(\frac{1}{2}\right)^{2 r} \sim \frac{1}{\sqrt{\pi r}}=\sqrt{\frac{2}{\pi(m-1)}} \quad \text { as } m \rightarrow \infty .
$$


2. Uniform bias. Let $U$ be uniform on the interval $(0,1)$. Then

$$
\alpha(i)=\int_{0}^{1}\left(\begin{array}{c}
2 r \\
r
\end{array}\right) u^{r}(1-u)^{r} d u=\frac{1}{m}, \quad \kappa(i)=\frac{1}{2}+\mathrm{o}(1) .
$$

3. Polarised bias. Let $\mathbb{P}\left(U=\frac{1}{3}\right)=\mathbb{P}\left(U=\frac{2}{3}\right)=\frac{1}{2}$. There exists $\gamma>0$ such that

$$
\alpha(i)=\mathrm{o}\left(e^{-\gamma m}\right), \quad \kappa(i)=\frac{1}{3}+\mathrm{o}(1) .
$$

We remind the reader that $f(m)=\mathrm{o}(g(m))$ means $f(m) / g(m) \rightarrow 0$ as $m \rightarrow \infty$. The success probabilities in Cases 1-3 follow by (2.5) from the above calculations.

Cases 2 and 3 are exemplars of more general situations in which: $\left(2^{\prime}\right)$ the distribution of $U$ on some neighbourhood of $\frac{1}{2}$ is absolutely continuous (see, for example, $\left[17\right.$, p. 674]), and $\left(3^{\prime}\right) U$ is almost surely bounded away from $\frac{1}{2}$ (see, for example, the formulation of [31, p. 592]).

Remark 2.4. Only in the case of independence does the absolute influence have the order of the square root $1 / \sqrt{m}$. In the two other situations above, the absolute influence is as small as $1 / m$ and $e^{-\gamma m}$, respectively. This illustrates the fragility of the square-root laws (1.1), (1.2) and their consequences for voting (see [34]).

Further discussion of the relationship between absolute and conditional influence may be found in [19, Sect. 2]. A review of influence for product measures is found at [25], see also [23, Sect. 4.5]. Uniform bias was discussed in [46], and polarised bias in [19]. Conditional influence is essentially the prediction value of [32].

2.4. Penrose's two square-root laws unified. We present next an elementary application of conditional influence (the proof is found at the end of the section). We will see its relevance in the discussion of the Penrose square-root laws in Remark 2.6 .

Proposition 2.5. Let $m=2 r+1$ be odd. Assume that $X$ and $-X$ have the same distributions, and let $A$ be the majority event of (2.6). Then $S=\sum_{i=1}^{m} X(i)$ satisfies

$$
\mathbb{E}|S|=\sum_{i=1}^{m} \kappa(i) .
$$

If $X$ is symmetric, then $\kappa=\kappa(i)$ is constant and $\mathbb{E}|S|=m \kappa$.

We next interpret Proposition 2.5 in the language of Penrose, see Section 1.2. Consider a population of size $N_{j}$, and suppose the corresponding vote-vector $X=$ $\left(X(1), X(2), \ldots, X\left(N_{j}\right)\right)$ is symmetric. Then $\kappa=\kappa(i)$ does not depend on $i$. The number $H$ of people voting 1 satisfies $H=\frac{1}{2}\left(S+N_{j}\right)$, so that, by Proposition 2.5 with $m=N_{j}$,

$$
\mathbb{E}\left|H-\frac{1}{2} N_{j}\right|=\frac{1}{2} N_{j} \kappa
$$


In conclusion, $\mathbb{E}\left|H-\frac{1}{2} N_{j}\right|$ grows in the manner of $\sqrt{N_{j}}$ if and only if $\kappa$ behaves in the manner of $1 / \sqrt{N_{j}}$.

Remark 2.6. In the language of Penrose [41], the mean 'edge' differs from the conditional influence by the constant multiple $N_{j}$. Thus, in the context of general distributions, conditional influence takes precedence over absolute influence. Penrose's argument implies that, in a two-tier voting system, the appropriate weight of state $j$ satisfies $w_{j} \propto N_{j} \kappa$, where $\kappa=\kappa_{j}$ is the conditional influence. In this sense, Penrose's "two" square-root laws are in reality only one, so long as one uses conditional rather than absolute influences: the asymptotic edge addressed by the second square-root law is simply a multiple of the asymptotic power addressed by the first square-root law. When voting is truly independent, the distinction between absolute and conditional influence is nominal only. Seen in the light of Remark 2.3, Proposition 2.5 supports the thesis that, for general probability measures, the success probability is a more central quantity than the absolute influence (cf. [36, Sect. 3.6]).

The question arises of deciding the 'correct' definition of influence in the general voting context. There does not seem to be a simple answer to this somewhat philosophical question, which lies beyond the scope of this mathematical paper. Some minor reflections are offered, within the context of the symmetric voting model of Definition 2.1.

(a) If we are trying to capture the probability that an individual can, as a theoretical exercise in free will, affect the outcome of a vote, then we might favour absolute influence. This interpretation requires stepping outside the mathematical model of Definition 2.1, by postulating the existence of a unique individual $\mathrm{P}$ who votes independently of the rest of the population. Then the absolute influence of $\mathrm{P}$ equals the probability that $\mathrm{P}$ 's vote is pivotal.

(b) When different votes are correlated, a sample of one vote contains information about the other votes. If we wish to gain such information, then conditional influence is a way to do so. On the other hand, conditional influence contains very little information about the power of any given individual. When votes are correlated, individual power tends to be rather small, and sometimes so small that it ceases to have great value as a discriminator.

(c) Only with the help of a probabilistic model can we calculate influence. An analysis of the above question depends on the interpretation of 'chance' or 'randomness' in such a model. Does it make practical sense to model votes as unbiased random variables? Possibly in response to this question, some authors have argued that the views of voters may not themselves be considered random, but it is rather the proposals that are random (see, for example, $[16$, p. 38] and [26, p. 360]). This interesting suggestion poses some philosophical 
challenges in its own right, not least arising from correlation between the responses of a given voter to different proposals.

Proof of Proposition 2.5. Let $1_{A}$ denote the indicator function of an event $A$. Then, since $X$ and $-X$ have the same distribution,

$$
\begin{aligned}
\mathbb{E}|S| & =\mathbb{E}\left(S 1_{S>0}\right)-\mathbb{E}\left(S 1_{S<0}\right) \\
& =2 \mathbb{E}\left(S 1_{S>0}\right) \\
& =2 \sum_{i=1}^{m} \mathbb{E}\left(X_{i} 1_{S>0}\right) \\
& =\sum_{i=1}^{m}\left[\mathbb{P}\left(S>0 \mid X_{i}=1\right)-\mathbb{P}\left(S>0 \mid X_{i}=-1\right)\right] \\
& =\sum_{i=1}^{m} \kappa(i) .
\end{aligned}
$$

Subject to symmetry, the constantness of $\kappa(i)$ holds by choosing suitable permutations of $\{1,2, \ldots, m\}$.

\section{TWO-TIER VOTING}

3.1. Two-tier voting systems. We assume there exist $s$ States with respective populations $N_{1}, N_{2}, \ldots, N_{s}$ (which we take for simplicity to be odd numbers). States are each allowed one representative on the Council of States. Each State is assumed to conduct a ballot on a given issue, and the vote of voter $i$ in State $j$ is denoted $X_{j}(i) \in\{-1,1\}$. The outcome of the vote in state $j$ is taken to be

$$
\chi_{j}:= \begin{cases}1 & \text { if } S_{j}:=\sum_{i=1}^{N_{j}} X_{j}(i)>0 \\ -1 & \text { otherwise }\end{cases}
$$

That is, $\frac{1}{2}\left(1+\chi_{j}\right)$ is the indicator function of the event that $S_{j}>0$.

Assumption $3.1([26])$. We assume the vectors $X_{j}=\left(X_{j}(i): i=1,2, \ldots, N_{j}\right)$, $j=1,2, \ldots, s$, are independent, which is to say that the votes of different States are independent. We make no assumption for the moment about the voters of any given State beyond that, for given $j$, the vectors $X_{j}$ are symmetric in that $X_{j}$ and $-X_{j}$ have the same distribution.

To the State $j$ is assigned a weight $w_{j}>0$, and we write $W=\sum_{j} w_{j}$ for the aggregate weight of the States. The representative of state $j$ votes $\chi_{j}$, and the 
weighted sum

$$
V:=\sum_{j=1}^{s} w_{j} \chi_{j}
$$

is calculated. The motion is said to pass if

$$
V>q W
$$

and to fail otherwise, where $q$ is a predetermined quota (this is not the quota of [51], but rather that of [26], see also (1.3)). This voting system depends on the weights $w=\left(w_{j}\right)$ and the quota $q$, and we refer to it as the $(w, q)$ system.

Since votes are assumed independent between States, the absolute and conditional influences coincide at the level of the Council.

Question 3.2. How should the weights $w_{j}$ and the quota $q$ be chosen?

We summarise two approaches.

3.2. Penrose/Kirsch and least squares [26, 41]. Penrose has argued that, within any given state, the strength of a vote is proportional to the mean 'edge', that is, the quantity $\mathbb{E}\left|N_{F}-N_{A}\right|$ where $N_{F}$ is the number voting for the successful outcome and $N_{A}$ is the number voting against. Now, $N_{F}-N_{A}=S_{j}$, where $S_{j}$ is given in (3.1). This motivates the 'Penrose' proposal that $w_{j}=\mathbb{E}\left|S_{j}\right|$.

Kirsch [26] has proposed choosing the $w_{j}$ in such a way as to minimise the mean sum of squared errors

$$
Q:=\mathbb{E}\left(\left[\sum_{j=1}^{s}\left(S_{j}-w_{j} \chi_{j}\right)\right]^{2}\right)
$$

A quick proof of the following proposition is given at the end of the subsection.

Proposition 3.3 ([26, Thm 2.1]). Subject to Assumption 3.1, the quantity $Q$ is minimised when $w_{j}=\mathbb{E}\left|S_{j}\right|$ for $j=1,2, \ldots, s$.

Thus, Kirsch's least-squares principle leads to the Penrose solution $w_{j}=\mathbb{E}\left|S_{j}\right|$, which we call the majority rule. As explained by Kirsch, this motivates the choice

$$
w_{j}= \begin{cases}\sqrt{N_{j}} & \text { if there is no long-range order } \\ N_{j} & \text { if there is long-range order }\end{cases}
$$


where 'long-range order' is interpreted as the non-decay of correlations (see the related Appendix B). ${ }^{1}$ For example, Case 1 of Section 2.3 has no long-range order, but Cases 2 and 3 possess long-range order. See also Remark 2.6.

Proof of Proposition 3.3. By Assumption 3.1,

$$
\begin{aligned}
Q & =\operatorname{var}\left(\sum_{j}\left(S_{j}-w_{j} \chi_{j}\right)\right) & & \text { since } \mathbb{E}\left(S_{j}\right)=\mathbb{E}\left(\chi_{j}\right)=0 \\
& =\sum_{j=1}^{s} \operatorname{var}\left(S_{j}-w_{j} \chi_{j}\right) & & \text { since the } X_{j} \text { are independent. }
\end{aligned}
$$

By calculus, the last summand is a minimum when $w_{j}=\mathbb{E}\left(S_{j} \chi_{j}\right)$ as claimed.

3.3. Słomczyński/Życzkowski and influence $[50,51,55]$. Let us concentrate on the situation in which the entire vote-set $\left(X_{j}(i): i=1,2, \ldots, N_{j}, j=1,2, \ldots, s\right)$ is a family of independent random variables. By independence, the absolute and conditional influences (within States) are equal. The influence $\alpha_{j}:=\alpha_{j}(i)$ of a member of State $j$ is (asymptotically as $N_{j} \rightarrow \infty$ ) proportional to $1 / \sqrt{N_{j}}$, by (2.8). The Penrose/Kirsch proposal of Section 3.2 amounts to $w_{j}=\mathbb{E}\left|S_{j}\right| \propto \sqrt{N_{j}}$. The product $\alpha_{j} w_{j}$ is (asymptotically, for large $N_{j}$ ) constant across the States. This may be seen as evidence that the voting system with this set of weights is 'fair' across the union of the States.

How does one calculate the so-called 'total influence' of a given voter in the $(w, q)$ system? A given voter is pivotal overall if $\mathrm{s} /$ he is pivotal within the relevant State vote, and furthermore the outcome of that vote is pivotal in the Council's vote. By Assumption 3.1, the total influence $I_{j}$ of voter $i$ in State $j$ is the product

$$
I_{j}=\alpha_{j} \beta_{j},
$$

where $\beta_{j}=\beta_{j}(w, q)$ is the influence of State $j$ in the Council's vote. (See [16, p. 67].) We seek a pair $(w, q)$ such that the total influences are equal (or nearly so) across the States $j$.

The total influences $I_{j}$ of (3.4) need not be proportional to the products $\alpha_{j} w_{j}$ of the previous paragraph, since the ratios $\beta_{j} / w_{j}$ are in general non-constant across the States. A number of authors including Słomczyński and Życzkowski [55] have developed the following approach.

1. Allocate to State $j$ the weight $w_{j}=\sqrt{N_{j}}$.

2. Calculate or estimate the State-influences $\beta_{j}$ as functions of $(w, q)$.

\footnotetext{
${ }^{1}$ The term 'long-range order' arises in statistical mechanics in situations where random spins $\sigma_{v}$ are located at the vertices $v$ of a lattice, such as the $d$-dimensional integer lattice $\mathbb{Z}^{d}$, and the correlation between two spins $\sigma_{v}, \sigma_{w}$ does not approach 0 as the distance between $v$ and $w$ diverges.
} 
3. Identify a quota $q$ such that $\beta_{j}$ is an approximately linear function of $w_{j}$.

4. The ensuing products $I_{j}=\alpha_{j} \beta_{j}$ are approximately constant across States.

They have proposed choosing the quota $q$ in (3.2) in such a way that, for the given weights $\left(w_{j}\right)$, the sum of squared differences

$$
Q:=\sum_{j=1}^{s}\left(\bar{w}_{j}-\bar{\beta}_{j}\right)^{2}
$$

is a minimum, where

$$
\bar{w}_{j}=\frac{100 w_{j}}{\sum_{k} w_{k}}, \quad \bar{\beta}_{j}=\frac{100 \beta_{j}}{\sum_{k} \beta_{k}}
$$

are the 'normalised' influences and weights, respectively (see Table 1). They present numerical, empirical, and theoretical evidence that this is often achieved when $q$ is near

$$
q^{*}:=\frac{\sqrt{N}}{\sum_{j} \sqrt{N_{j}}}, \quad \text { where } N=\sum_{j=1}^{s} N_{j} .
$$

The theoretical foundation for this proposal lies in: (i) approximating $\beta_{j}$ by a Gaussian integral, and (ii) picking $q$ such that the integrand is close to linear in $w_{j}$. The latter step is achieved by finding the point at which the $N\left(\mu, \sigma^{2}\right)$ Gaussian density function has an inflection, and is thus locally closest to being locally linear. This inflection is easily found by calculus to be at $q:=\mu \pm \sigma$, and this leads to the formula (3.5).

In summary, they argue that, when $w_{j}=\sqrt{N_{j}}$ and $q=q^{*}$, the $\beta_{j}=\beta_{j}(w, q)$ are close to the $w_{j}$, and hence the total influences $I_{j}=\alpha_{j} \beta_{j}$ are close to the products $\alpha_{j} w_{j}$. Finally, since $\alpha_{j} \sim C / \sqrt{N_{j}}$ and $w_{j}=\sqrt{N_{j}}$, the last product is asymptotically constant across the States.

The above procedure is termed the Jagiellonian Compromise (or JagCom). We discuss in Section 4 some aspects of the derivation of the quota $q^{*}$ in (3.5). Some peripheral support for this choice of quota may be found in the analyses of "toy models' in [51, 53].

Remark 3.4. The weights $w_{j}$ are chosen first in the JagCom, and then the quota $q$ is chosen according to a linearisation argument. It may instead be preferable to choose the parameters $(w, q)$ in such a way that the deviation in the total influences $I_{j}$ is a minimum. See, for example, [33].

\section{4. 'TOTAL InfluenCES' IN A TWO-TIER SYSTEM}

4.1. Total influences. A mathematical derivation of the JagCom quota $q^{*},(3.5)$, seems to require certain approximations which we discuss next. The first issue is to 
identify the purpose of the analysis. Let $I_{j}$ be the total influence of a member of State $j$, as in (3.4). One extreme way of achieving the near-equality of the $I_{j}$ is to set the quota $q$ on the left side of (1.3) to be either $-\epsilon+\sum_{j} \sqrt{N_{j}}$ or its negation, where $\epsilon>0$ is small. If we insist on such unanimity, we achieve

$$
I_{j}=\alpha_{j}\left(\frac{1}{2}\right)^{s} \sim \frac{C}{\sqrt{N_{j}}}\left(\frac{1}{2}\right)^{s} .
$$

For large $s$, these influences are nearly equal, indeed nearly equal to 0 . Their ratios however can be as large as $\sqrt{N_{\max } / N_{\min }}$, where $N_{\max }$ (respectively, $N_{\min }$ ) is the maximum (respectively, minimum) population size. An alternative target is that the ratios $I_{j} / I_{k}$ be as close to unity as possible, and a secondary target might be that the total influences are as large as possible. We consider this next.

Consider a vote of the Council in which each State $k$ has a preassigned weight $w_{k}>0$. Let $j \in\{1,2, \ldots, s\}$. By (3.2), State $j$ is pivotal for the outcome if: the set $J$ of States (other than $j$ ) voting for the motion is such that

$$
w_{J}+w_{j}-w_{\bar{J}}>q W, \quad w_{J}-w_{j}-w_{\bar{J}} \leq q W,
$$

where $J \subseteq\{1,2, \ldots, s\} \backslash\{j\}, \bar{J}=\{1,2, \ldots, s\} \backslash(J \cup\{j\})$, and

$$
w_{K}:=\sum_{k \in K} w_{k}, \quad K \subseteq\{1,2, \ldots, s\} .
$$

Inequalities (4.1) may be written in the form $q W-w_{j}<Z_{j} \leq q W+w_{j}$ where

$$
Z_{j}=w_{J}-w_{\bar{J}}=\sum_{k \neq j} w_{k} \chi_{k}, \quad j \in\{1,2, \ldots, s\},
$$

and $\left(\chi_{k}: k=1,2, \ldots, s\right)$ is a family of independent Bernoulli random variables with

$$
\mathbb{P}\left(\chi_{k}=1\right)=\mathbb{P}\left(\chi_{k}=-1\right)=\frac{1}{2} .
$$

Therefore, State $j$ is pivotal in the Council with probability

$$
\begin{aligned}
\beta_{j} & :=\mathbb{P}\left(q W-w_{j}<Z_{j} \leq q W+w_{j}\right) \\
& =F_{Z_{j}}\left(q W+w_{j}\right)-F_{Z_{j}}\left(q W-w_{j}\right),
\end{aligned}
$$

where $F_{Z_{j}}$ is the distribution function of $Z_{j}$. (Similar formulae appear in [51, App.].)

Słomczyński and Życzkowski [55] argue that the $F_{Z_{j}}$ are 'nearly' Gaussian, and they consider the appoximation

$$
\beta_{j} \approx 2 w_{j} \phi_{\mu_{j}, \sigma_{j}}(q W)
$$

where $\phi_{\mu, \sigma}$ is the $N\left(\mu, \sigma^{2}\right)$ Gaussian density function, and

$$
\mu_{j}=\mathbb{E}\left(Z_{j}\right)=0, \quad \sigma_{j}^{2}=\operatorname{var}\left(Z_{j}\right)=\sum_{k \neq j} w_{k}^{2} .
$$


More precisely, (4.4) expresses the hypothesis that the distributions are nearly Gaussian, and that the approximation is sufficiently uniform to be accurate to the first order, in terms of the density function. They argue that the approximation is most accurate when $q$ is chosen in such a way that $q W$ is a point of inflection of $\phi_{\mu_{j}, \sigma_{j}}$, and this leads to the choice $q=q^{*}$, with $q^{*}$ as in (3.5). It is explained at the end of Appendix C, to which the reader is referred for further details, that the ensuing approximations are good in the case of the population-sizes of the EU Member States, but no proof is known to sufficient accuracy to permit the rigorous deduction of the quota, $q^{*}$. The currently best theoretical tool for Gaussian approximations is the so-called Berry-Esseen bound, and this is not good enough for our purpose here. See Table 2.

4.2. The argument via numerical methods. Once one has accepted the thesis that voters are independent and unbiased, there is a transparent logic to the choice of weights $w_{j}=\sqrt{N_{j}}$. Attention then turns to the choice of quota $q$. It is shown in Appendix $\mathrm{C}$ that the mathematical argument of Słomczyński and Życzkowski [51], while neat, is at best incomplete. In contrast, the numerical evidence of [49], in favour of $q=q^{*}$, retains some persuasive power. Similar numerical work has been carried out for the current article using QMV2017 population data taken from [44], with the results reported in Table 1 . These results are exact rather than being based on simulation.

Table 1 lends some support to the choice $q=q^{*}$.

(a) The ratios of normalised influences $\bar{\beta}_{j}$ to normalised weights $\bar{w}_{j}$ are very close to 1 when $q=q^{*}$.

(b) Further calculations show that the sum of squared differences $Q=\sum_{j}\left(\bar{w}_{j}-\right.$ $\left.\bar{\beta}_{j}\right)^{2}$, considered as a function of $q=0, \frac{1}{2} q^{*}, q^{*}, \frac{3}{2} q^{*}$, is a minimum when $q=q^{*}$. (More refined calculations are possible.)

We note, however, the following.

(i) The choice $q=q^{*}$ lacks transparency. In contrast, the choice $q=0$ is simple and easy to explain.

(ii) The ratios $\bar{\beta}_{j} / \bar{w}_{j}$ are also close to 1 when $q=0$. The agreement is not quite so perfect as when $q=q^{*}$, but the differences are minor.

(iii) The sum $Q$ is similarly close to 0 when $q=0$, albeit not so close as when $q=q^{*}$.

(iv) The influences $\beta_{j}$ are largest when $q=0$. (See also [51, App.].)

In summary, the numerics are best when $q=q^{*}$, but the improvements relative to the more transparent choice of $q=0$ are minor. The numerical differences between these two cases (and indeed other reasonable values of $q$ ) are so small that they are 


\begin{tabular}{|c|c|c|c|c|c|c|c|c|c|}
\hline \multirow{2}{*}{\multicolumn{2}{|c|}{$\begin{array}{l}\text { Member State } \\
j \mid\end{array}$}} & \multicolumn{2}{|c|}{ weights } & \multicolumn{3}{|c|}{$q=0$} & \multicolumn{3}{|c|}{$q=q^{*}$} \\
\hline & & $w_{j}$ & $\bar{w}_{j}$ & $\beta_{j}$ & $\bar{\beta}_{j}$ & $\bar{\beta}_{j} / \bar{w}_{j}$ & $\beta_{j}$ & $\bar{\beta}_{j}$ & $\bar{\beta}_{j} / \bar{w}_{j}$ \\
\hline 1 & Germany & 9.059 & 9.963 & 0.357 & 10.414 & 1.045 & 0.211 & 9.937 & 0.997 \\
\hline 2 & France & 8.165 & 8.979 & 0.317 & 9.239 & 1.029 & 0.191 & 8.984 & 1.001 \\
\hline 3 & Italy & 7.830 & 8.611 & 0.302 & 8.816 & 1.024 & 0.183 & 8.619 & 1.001 \\
\hline 4 & Spain & 6.815 & 7.495 & 0.260 & 7.575 & 1.011 & 0.159 & 7.507 & 1.002 \\
\hline 5 & Poland & 6.162 & 6.777 & 0.233 & 6.802 & 1.004 & 0.144 & 6.787 & 1.001 \\
\hline 6 & Romania & 4.445 & 4.888 & 0.166 & 4.839 & 0.990 & 0.104 & 4.891 & 1.001 \\
\hline 7 & Netherlands & 4.152 & 4.566 & 0.155 & 4.512 & 0.988 & 0.097 & 4.568 & 1.000 \\
\hline 8 & Belgium & 3.360 & 3.695 & 0.125 & 3.636 & 0.984 & 0.078 & 3.696 & 1.000 \\
\hline 9 & Greece & 3.285 & 3.613 & 0.122 & 3.554 & 0.984 & 0.077 & 3.613 & 1.000 \\
\hline 10 & Czech Rep. & 3.232 & 3.554 & 0.120 & 3.495 & 0.983 & 0.075 & 3.554 & 1.000 \\
\hline 11 & Portugal & 3.216 & 3.537 & 0.119 & 3.478 & 0.983 & 0.075 & 3.537 & 1.000 \\
\hline 12 & Sweden & 3.162 & 3.477 & 0.117 & 3.418 & 0.983 & 0.074 & 3.477 & 1.000 \\
\hline 13 & Hungary & 3.135 & 3.448 & 0.116 & 3.389 & 0.983 & 0.073 & 3.447 & 1.000 \\
\hline 14 & Austria & 2.952 & 3.246 & 0.109 & 3.189 & 0.982 & 0.069 & 3.246 & 1.000 \\
\hline 15 & Bulgaria & 2.675 & 2.942 & 0.099 & 2.886 & 0.981 & 0.062 & 2.941 & 1.000 \\
\hline 16 & Denmark & 2.388 & 2.626 & 0.088 & 2.574 & 0.980 & 0.056 & 2.625 & 1.000 \\
\hline 17 & Finland & 2.338 & 2.571 & 0.086 & 2.520 & 0.980 & 0.055 & 2.570 & 1.000 \\
\hline 18 & Slovakia & 2.326 & 2.558 & 0.086 & 2.507 & 0.980 & 0.054 & 2.557 & 1.000 \\
\hline 19 & Ireland & 2.160 & 2.375 & 0.080 & 2.327 & 0.980 & 0.050 & 2.374 & 1.000 \\
\hline 20 & Croatia & 2.047 & 2.251 & 0.076 & 2.204 & 0.979 & 0.048 & 2.250 & 1.000 \\
\hline 21 & Lithuania & 1.700 & 1.870 & 0.063 & 1.829 & 0.978 & 0.040 & 1.868 & 0.999 \\
\hline 22 & Slovenia & 1.437 & 1.580 & 0.053 & 1.545 & 0.978 & 0.034 & 1.579 & 0.999 \\
\hline 23 & Latvia & 1.403 & 1.543 & 0.052 & 1.508 & 0.977 & 0.033 & 1.542 & 0.999 \\
\hline 24 & Estonia & 1.147 & 1.261 & 0.042 & 1.233 & 0.978 & 0.027 & 1.260 & 0.999 \\
\hline 25 & Cyprus & 0.921 & 1.013 & 0.034 & 0.990 & 0.977 & 0.021 & 1.012 & 0.999 \\
\hline 26 & Luxembourg & 0.759 & 0.835 & 0.028 & 0.815 & 0.976 & 0.018 & 0.834 & 0.999 \\
\hline \multirow[t]{2}{*}{27} & Malta & 0.659 & 0.725 & 0.024 & 0.708 & 0.977 & 0.015 & 0.724 & 0.999 \\
\hline & Totals & 90.930 & 100 & 3.429 & 100 & & 2.123 & 100 & \\
\hline
\end{tabular}

TABLE 1. Member State $j$ has weight $w_{j}=\sqrt{N_{j}}$ and normalised weight $\bar{w}_{j}=100 w_{j} / W$, where $W=\sum_{j} w_{j}$. Two values of the quota $q$ are considered, namely, $q=0$ and $q=q^{*}$ (see (3.5)). For each, the influences $\beta_{j}$ have been computed, and the normalised influences $\bar{\beta}_{j}=100 \beta_{j} / B$ are given above, where $B=\sum_{j} \beta_{j}$. The ratios $\bar{\beta}_{j} / \bar{w}_{j}$ are presented alongside the $\bar{\beta}_{j}$. The ratios lie in the interval $[0.976,1.045]$ when $q=0$, and in the interval $[0.997,1.002]$ when $q=q^{*}$. 
unlikely to be separated by any technical analysis of the type of Appendix C. (See also [54, Fig. 7].) We conclude the following.

1. On the basis of the theoretical and numerical evidence concerning the ratios $\bar{\beta}_{j} / \bar{w}_{j}$, there is no convincing evidence that any one value of the quota is materially preferable to any other. ${ }^{2}$

2. The total influences $I_{j}$ are largest when $q=0$.

\section{Some Remarks on the Jagiellonian Compromise}

Theoreticians propose, politicians dispose (and certain Presidents of the United States have historically played on both teams). Members of each group have interests and potential conflicts. The theoretician earns respect through honest assessment of the virtues (or not) of, and principles underlying, a particular proposal. They hope that politicians will accord fair weight and balance to principled proposals irrespective of personal advantage. While theoreticians are usually free of conflicts arising out of employment within a politically aligned organization, politicians are usually heavily conflicted (see, for example, [44]).

Communication between the two groups can be challenging. The use of language such as 'local limit theorem' and 'Berry-Esseen bound' tends to create barriers. Such methodology is however key to proper study of the two-tier voting system of Sections 3-4, and practitioners have worked diligently to communicate its relevance.

The JagCom proposes the use of square-root weights $w_{j}=\sqrt{N_{j}}$ with a specific choice of the quota $q$. Each of these two proposals will rightly continue to attract critical discussion.

The square-root weights of equation (1.1) and Proposition 3.3 may be justified if: (i) there is no bias, and (ii) there is no 'long-range order' (in the language of statistical mechanics). Each of these assumptions seems over-perfectionist. Issues before the Council may be systematically more popular in some States than in others, and the consequent biases risk undermining either or both of the above two assumptions. The 'collective bias' model of Kirsch and others (see Section 2) is both more flexible and more empirical, at serious cost to the square-root laws for influence and majority (see [29]). Other authors have considered the effect on weights of introducing a concave utility function (see Koriyama et al. [31]). Such approaches give rise to weight distributions which, in turn, benefit from calibration against the politics and practical workings of the Council. The right choice of weights is not a simple matter of finding some neat mathematics. That said, no concrete proposal to displace squareroot weights in the JagCom is made in the current work.

\footnotetext{
${ }^{2}$ Large positive or negative values are evidently poor, but we consider here only values $q$ such that $q W / \sqrt{N}$ has order 1 . Other choices for $q$ have been considered in, for example, [10, 13].
} 
Having chosen the weights, the identification of the quota is subject to similar ambiguities. The JagCom 'exact' quota $q^{*}$ of (3.5) hinges on the assumptions of square-root weights and equality of absolute influence, in combination with numerical data and the Gaussian approximation of Appendix C. The last is unproven in the current context of the QMV2017 population data of the States of the EU.

In their favour, the proposed square-root weights and the exact quota $q^{*}$ of the JagCom have been derived via a set of principles that can be stated unambiguously and analysed fairly rigorously, and which are robust with respect to changes in population data. Proponents argue that they are thus less susceptible than many alternatives to 'political meddling'.

We turn now to the numerics of the JagCom quota $q^{*}$. If the ratios $\bar{\beta}_{j} / \bar{w}_{j}$ in Table 1 are close to 1 , then the total influences $I_{j}=\alpha_{j} \beta_{j}$ of (3.4) are almost constant across Member States. As indicated in the shaded columns of the table, this holds for both $q=0$ and $q=q^{*}$ (they are nearly perfect when $q=q^{*}$, and very close for other values of $q$ ). Indeed this holds for a range of values of $q$ including the values 0 and $q^{*}$ (see also [54]). One may deduce that, from a practical point of view, there is little to choose between different values of $q$. This may be a situation in which political considerations may have the final word. It seems generally considered to be the case that there is no enthusiasm amongst politicians for the choice $q=0$, on the grounds that a body such as the EU Council should seek a compromise between simple majority and unanimity.

Overall, the details of the JagCom rely on a number of assumptions that are arguably fragile and/or unrealistic. This potential weakness needs to be acknowledged when making the case for the JagCom. The JagCom is a legitimate proposal for the two-tier voting system of the Council of the EU, whose finer details may profit from input by politicians in choosing a system judged to serve well the needs of the nearly 450 million residents of the 27 Member States of the European Union without the United Kingdom. Our closing quote (Machover [38, Abs.]) accords a balanced responsibility to both theoreticians and politicians: "This is essentially a political matter; but a political decision ought to be made in a theoretically enlightened way."

\section{Appendix A. Circular voting}

In Definition 2.1, condition (ii) is weaker than requiring that $X$ be exchangeable (see [24, p. 324]). Here is a simple one-dimensional example of a random vector that is symmetric but not exchangeable. Suppose the $m(\geq 4)$ voters are distributed evenly around a circular table. Let $Z_{1}, Z_{2}, \ldots, Z_{m}$ be the outcomes of $m$ independent tosses of a fair coin that shows the values \pm 1 . Let $X(i)$ be the majority value of $Z_{i-1}$, $Z_{i}, Z_{i+1}$, with the convention that $Z_{m+k}=Z_{k}$ for all $k$ (and a similar convention for the $X(i))$. It may be checked that $X(i)$ and $X(j)$ are independent if and only if $i$ 
and $j$ are distance 3 or more away from each other. The joint distribution of $X$ is invariant under the rotation $i \mapsto i+1$ modulo $m$, and is hence symmetric.

Similar examples may be constructed in two and higher dimensions. In models that incorporate a spatial element in the relationships between individual voters, symmetry may be a reasonable assumption when exchangeability is not.

\section{Appendix B. 'Ferromagnetic voting'}

Kirsch [26] proposed studying the voting problem via the analogy of a ferromagnetic model, such as the classical Ising model. He concentrated on the so-called Curie-Weiss (or mean-field) model, in which each vertex $v$ of the complete graph has a random spin $\sigma_{v}$ taking values in $\{-1,1\}$ according to a certain probability distribution dictated by the so-called Ising model. The reader is referred to his paper for further details.

The analysis is especially simple in this so-called 'mean-field' case since the complete graph has the maximum of symmetry. The mean-field case is, in a sense familiar to mathematical physicists, an infinite-dimensional model. We note that similar results are fairly immediate for the more pertinent finite-dimensional systems also, as follows. For concreteness, let $d \geq 1$ and let $\mathbb{T}_{n}$ be the $d$-dimensional torus obtained from the square grid $\{0,1, \ldots, \bar{n}\}^{d}$ with periodic boundary conditions. Let $\beta_{\mathrm{c}}$ be the critical value of the inverse-temperature $\beta$ of the Ising model on $\mathbb{Z}^{d}$ (we refer to $[22,23]$ for explanations of the model and notation). Interpreting $\sigma_{v}$ as the vote of an individual placed at the vertex $v$, the aggregate vote

$$
S=\sum_{v \in \mathbb{T}_{n}} \sigma_{v}
$$

satisfies

$$
\mathbb{E}|S| \approx \begin{cases}n^{d / 2} & \text { if } \beta<\beta_{\mathrm{c}} \\ n^{d} & \text { if } \beta>\beta_{\mathrm{c}} .\end{cases}
$$

The bibliography associated with the Ising model and its ramifications is extended and complex, and is directed mostly at the corresponding infinite-volume problem defined on the entire $d$-dimensional space $\mathbb{Z}^{d}$. Some of the above claims for periodic boundary conditions are well known, and others may be derived from classical results. The relevant literature includes [1, 14, 40].

\section{Appendix C. Gaussian approximation}

Słomczyński and Życzkowski argue that the distribution function $F_{Z_{j}}$ in (4.3) is 'roughly' Gaussian with mean and variance given by (4.5). Motivated by the local central limit theorem for non-identically distributed random variables (see [24, p. 
195] and [18], or otherwise), we aspire to a Gaussian approximation of (4.3) of the form

$$
\begin{aligned}
\beta_{j} & \approx \int_{q W-w_{j}}^{q W+w_{j}} \phi_{\sigma_{j}}(z) d z \approx 2 w_{j} \phi_{\sigma_{j}}(q W) \\
& =\frac{2 w_{j}}{\sqrt{2 \pi \sigma_{j}^{2}}} \exp \left(-\frac{(q W)^{2}}{2 \sigma_{j}^{2}}\right)
\end{aligned}
$$

where $\phi_{\sigma}$ is the density function of the $N\left(0, \sigma^{2}\right)$ Gaussian distribution. (No sufficiently accurate estimate of the error in the first approximation of (C.1) is currently available.) This leads to the following approximation for the total influence of a voter in State $j$ :

$$
I_{j}=\alpha_{j} \beta_{j} \simeq \frac{C}{\sqrt{N_{j}}} \frac{2 w_{j}}{\sqrt{\Delta^{2}-w_{j}^{2}}} \exp \left(-\frac{1}{2} \cdot \frac{(q W)^{2}}{\left(\Delta^{2}-w_{j}^{2}\right)}\right),
$$

where $C>0$ is an absolute constant, and

$$
\Delta^{2}=\sum_{k=1}^{s} w_{k}^{2}
$$

Let $\delta=N_{\max } / N$, and set $w_{j}=\sqrt{N_{j}}$. (A similar anaysis is valid with $w_{j}$ set to the mean edge of Remark 1.1.)

(a) If we set $q=q^{*}$ as in (3.5), we obtain the approximate inequalities

$$
\frac{2 C}{\Delta} e^{-1 /[2(1-\delta)]} \preceq I_{j}=\alpha_{j} \beta_{j} \preceq \frac{2 C}{\Delta \sqrt{1-\delta}} e^{-1 / 2}, \quad j=1,2, \ldots, s,
$$

with $\Delta=\sqrt{N}$. (The symbol $\preceq$ is used in order to indicate that the inequalities are based on the unquantified approximation (C.1).) These bounds are independent of the choice of $j$, and are increasingly close to one another in the limit as $\delta \rightarrow 0$.

(b) If, instead, we set $q=0$, we obtain the inequalities (C.2) with the exponential terms removed.

The exact numerical values of the $\beta_{j}$ are calculated in Section 4.2 for the particular case of the 27 Member States of the European Union post-Brexit.

The above analysis depends on two Assumptions:

1. the normal (or Gaussian) approximation (C.1) is reasonable,

2. the ratio $\delta=N_{\max } / N$ is small.

Assumption 2 is unavoidable in some form, and its use within (C.2) is quantified therein. We therefore concentrate henceforth on Assumption 1. The approximation 
of (C.1) is a statement about a finite population, and thus one needs a rate of convergence in the central limit theorem. The classical such result is as follows.

Theorem C.1 (Berry-Esseen [7, 15, 48]). There exists $C \in[0.4906,0.5600]$ such that the following holds. Let $X_{1}, X_{2}, \ldots, X_{s}$ be independent random variables with

$$
\mathbb{E}\left(X_{i}\right)=0, \quad \mathbb{E}\left(X_{i}^{2}\right)=t_{i}^{2}>0, \quad \mathbb{E}\left(\left|X_{i}\right|^{3}\right)=\gamma_{i}<\infty,
$$

and write

$$
\sigma^{2}=\sum_{i=1}^{s} t_{i}^{2}, \quad S=\frac{1}{\sigma} \sum_{i=1}^{s} X_{i}
$$

Then

$$
\sup _{z \in \mathbb{R}}|\mathbb{P}(S \leq z)-\Phi(z)| \leq \frac{C}{\sigma^{3}} \sum_{i} \gamma_{i},
$$

where $\Phi$ is the distribution function of the $N(0,1)$ distribution.

Applying this to the random variable $Z_{j}$ of $(4.2)$, we obtain

$$
\begin{aligned}
\sup _{z \in \mathbb{R}}\left|F_{Z_{j}}(z)-\Phi_{\sigma_{j}}(z)\right| & =\sup _{z \in \mathbb{R}}\left|\mathbb{P}\left(Z_{j} / \sigma_{j} \leq z\right)-\Phi(z)\right| \\
& \leq C \frac{\sum_{k \neq j} w_{k}^{3}}{\left(\sum_{k \neq j} w_{k}^{2}\right)^{3 / 2}},
\end{aligned}
$$

where $\sigma_{j}^{2}$ is given in (4.5), and $\Phi_{\sigma}$ is the distribution function of the $N\left(0, \sigma^{2}\right)$ distribution. Therefore, by (4.3) (see (C.1)),

$$
\left|\beta_{j}-\int_{q W-w_{j}}^{q W+w_{j}} \phi_{\sigma_{j}}(z) d z\right| \leq 2 C \frac{\sum_{k \neq j} w_{k}^{3}}{\left(\sum_{k \neq j} w_{k}^{2}\right)^{3 / 2}}
$$

where $2 C \leq 1.12$.

Example C.2 (EU27). Suppose $s=27$ and the State populations $N_{1}, N_{2}, \ldots, N_{27}$ are the QMV2017 figures for the Member States of the EU, as in [44, Table 1]. We write $N_{1}>N_{2}>\cdots>N_{27}$, so that $N_{\max }=N_{1}$, and we choose $w_{j}=\sqrt{N_{j}}$ and $q=q^{*}$ with $q^{*}$ as in (3.5).

The integral on the left side of (C.4) may be expressed as

$$
\int_{\left(\sqrt{N}-w_{j}\right) / \sqrt{N-N_{j}}}^{\left(\sqrt{N}+w_{j}\right) / \sqrt{N-N_{j}}} \phi(z) d z
$$

where $\phi=\phi_{0}$. Its numerical value decreases monotonically from 0.207 (when $j=1$ ) to 0.015 (when $j=27$ ). The Berry-Esseen bound on the right side of (C.4) takes the value 0.332 (when $j=1$ ), 0.349 (when $j=5$ ), and 0.334 (when $j=27$ ), and 
ON INFLUENCE AND COMPROMISE

\begin{tabular}{l|l||lcc|ccc}
\multicolumn{3}{l||}{ Member State } & \multicolumn{3}{c|}{$q$} & \multicolumn{3}{c}{$q=q^{*}$} \\
$j$ & & exact & JagCom & BE interval & exact & JagCom & BE interval \\
\hline 1 & Germany & 0.357 & 0.379 & {$[0.03,0.70]$} & 0.211 & 0.205 & {$[-0.13,0.54]$} \\
3 & Italy & 0.302 & 0.319 & {$[-0.03,0.65]$} & 0.183 & 0.178 & {$[-0.17,0.52]$} \\
5 & Poland & 0.233 & 0.244 & {$[-0.11,0.59]$} & 0.144 & 0.141 & {$[-0.21,0.49]$} \\
7 & Netherlands & 0.155 & 0.160 & {$[-0.19,0.50]$} & 0.097 & 0.095 & {$[-0.25,0.44]$} \\
9 & Greece & 0.122 & 0.126 & {$[-0.22,0.47]$} & 0.077 & 0.075 & {$[-0.27,0.42]$} \\
\hline 11 & Portugal & 0.119 & 0.123 & {$[-0.22,0.46]$} & 0.075 & 0.074 & {$[-0.27,0.41]$} \\
13 & Hungary & 0.116 & 0.120 & {$[-0.22,0.46]$} & 0.073 & 0.072 & {$[-0.27,0.41]$} \\
15 & Bulgaria & 0.099 & 0.102 & {$[-0.24,0.44]$} & 0.062 & 0.061 & {$[-0.28,0.40]$} \\
17 & Finland & 0.086 & 0.089 & {$[-0.25,0.43]$} & 0.055 & 0.054 & {$[-0.28,0.39]$} \\
19 & Ireland & 0.080 & 0.082 & {$[-0.26,0.42]$} & 0.050 & 0.050 & {$[-0.29,0.39]$} \\
\hline 21 & Lithuania & 0.063 & 0.064 & {$[-0.27,0.40]$} & 0.040 & 0.039 & {$[-0.30,0.37]$} \\
23 & Latvia & 0.052 & 0.053 & {$[-0.28,0.39]$} & 0.033 & 0.032 & {$[-0.30,0.37]$} \\
25 & Cyprus & 0.034 & 0.035 & {$[-0.30,0.37]$} & 0.021 & 0.021 & {$[-0.31,0.36]$} \\
27 & Malta & 0.024 & 0.025 & {$[-0.31,0.36]$} & 0.015 & 0.015 & {$[-0.32,0.35]$} \\
\hline
\end{tabular}

TABLE 2. Numerical data for odd values of $j$ and the quotas $q=0$ and $q^{*}$. Three values are given in each case: the exact value of the Stateinfluence $\beta_{j}$ as in Table 1 (labelled exact), the approximation (4.4) (labelled JagCom), and the rigorous interval for the State-influence calculated by the Berry-Esseen bound (C.4) (labelled BE interval). Note the close agreement between the exact values and the JagCom approximations in all cases, and especially so when $q=q^{*}$. The rigorous Berry-Esseen bound is insufficient to rule out even $\beta_{j}=0$ except in the unique case $q=q^{*}, j=1$. The calculations involving the Gaussian distribution have been performed using Microsoft Excel.

is monotone on each of the two intervals $j \in[1,5]$ and $j \in[5,27]$. The bounds are too large to yield useful information about the $\beta_{j}$, and thus they cannot be estimated using the Berry-Esseen bound. In contrast, the values of the integral in (C.5) are notably close to the exact values of Table 1. A similar analysis is valid when $q=0$. This numerical information is summarised in Table 2.

We emphasise that the above observations do not invalidate the JagCom. Preferable to the Berry-Esseen bound would be a sufficiently precise rate of convergence in the local central limit theorem for discrete, non-identically distributed random variables. We are unaware of such a result. 
We return to the question of the accuracy of the two approximations in (C.1) (see also (4.4)). Although this has not been investigated systematically in the current work, exploratory numerical calculations have been performed with a Union of 20 States, in which $N_{\max } / N$ is approximately that of EU27 with QMV2017. All such calculations indicate that the left side of (C.4) is only rarely greater than $10^{-2}$, and this is positive evidence for the Gaussian approximation with general population figures comparable to those of EU27.

The approximation (4.4) amounts to a further approximation around $q^{*}$, based upon the near-linearity of the Gaussian density function near a point of inflection. The empirical evidence indicates, as above, that the discrepancy is generally less than $10^{-2}$.

We conclude this appendix as follows. No mathematical proof is known of the optimality of the choice (3.5) of the quota $q^{*}$ in the JagCom for general population distributions. Even if a rate can be proved in the appropriate central limit theorem, it is unlikely to be sufficiently tight to justify the choice $q^{*}$. Numerical data is, however, positive.

\section{ACKNOWLEDGEMENTS}

This work was supported in part by the Engineering and Physical Sciences Research Council under grant EP/I03372X/1. It was conducted partly during a visit to the Statistics Department of the University of California at Berkeley, and was completed during a visit to Keio University, Japan. Friedrich Pukelsheim has kindly made a number of valuable suggestions for this work, and has provided some further references. Aernout van Enter has advised on the bibliography of the Ising model. The author thanks Wojciech Słomczyński for his comments on the history of the JagCom and for drawing his attention to a number of additional references. Thanks are due to the three referees, for their careful readings and their insightful and constructive comments.

\section{REFERENCES}

[1] M. Aizenman, H. Duminil-Copin, and V. Sidoravicius, Random currents and continuity of Ising model's spontaneous magnetization, Commun. Math. Phys. 334 (2015), 719-742.

[2] N. Alon and P. H. Edelman, The inverse Banzhaf problem, Social Choice and Welfare 34 (2010), 371-377.

[3] M. L. Balinski and H. P. Young, Fair Representation, Yale University Press, New Haven, Conn., 1982.

[4] J. F. Banzhaf, Weighted voting doesn't work: a mathematical analysis, Rutgers Law Review 13 (1965), 317-343.

[5] R. N. Barlow and F. Proschan, Mathematical Theory of Reliability, Wiley, New York, 1965.

[6] M. Ben-Or and N. Linial, Collective coin flipping, Randomness and Computation, Academic Press, New York, 1990, pp. 91-115. 
[7] A. C. Berry, The accuracy of the Gaussian approximation to the sum of independent variates, Trans. Amer. Math. Soc. 49 (1941), 122-136.

[8] J. M. Bilbao and 14 others, How to vote in the Council of the European Union? Open letter, (3 July 2008), http://chaos.if.uj.edu.pl/ karol/pdf/voting08.pdf.

[9] J. M. Bilbao and 46 others, Letter to the Governments of the EU Member States. Open letter, (27 April 2004), https://www.wahlrecht.de/news/2004/12-openletter.pdf, http:// chaos.if.uj.edu.pl/ karol/pdf/OtwartyList.pdf.

[10] O. Birkmeier, Machtindizes und Fairness-Kriterien in gewichteten Abstimmungssystemen mit Enthaltungen, vol. 18, Augsburger Schriften für Mathematik, Physik und Informatik, 2011.

[11] O. Birkmeier and F. Pukelsheim, A probabilistic re-view of Felsenthal and Machover's "The Measurement of Voting Power", Voting Powers and Procedures (R. Fara, D. Leech, and M. Salles, eds.), Springer Verlag, Cham, 2014, pp. 117-124.

[12] E. Cartlidge, There's a fairer way to allot seats in the European Parliament, mathematicians say - but politicians don't like it, Science (8 March 2018), http://www.statslab.cam.ac. $\mathrm{uk} /$ grg/PG-Science2018.html.

[13] P.-L. Chang, V. C. H. Chua, and M. Machover, L. S. Penrose's limit theorem: Tests by simulation, Math. Soc. Sci. 51 (2006), 90-106.

[14] J. T. Cox and G. R. Grimmett, Central limit theorems for associated random variables and the percolation model, Ann. Probab. 12 (1984), 514-528.

[15] C.-G. Esseen, On the Liapunoff limit of error in the theory of probability, Arkiv Mat. Astr. Fys. A28 (1942), 1-19.

[16] D. Felsenthal and M. Machover, The Measurement of Power: Theory and Practice, Problems and Paradoxes, Edward Elgar, Cheltenham, UK, 1998.

[17] A. Gelman, J. N. Katz, and J. Bafumi, Standard voting power indexes do not work: An empirical analysis, Brit. J. Polit. Sci. 34 (2004), 657-674.

[18] R. Giuliano and M. Weber, Approximate local limit theorems with effective rate and application to random walks in random scenery, Bernoulli 23 (2017), 3268-3310.

[19] B. T. Graham and G. R. Grimmett, Influence and sharp threshold theorems for monotonic measures, Ann. Probab. 34 (2006), 1726-1745.

[20] _ Sharp thresholds for the random-cluster and Ising models, Ann. Appl. Probab. 21 (2011), 240-265.

[21] G. R. Grimmett, Percolation, Springer Verlag, Berlin, 1999.

[22] _ The Random-Cluster Model, Springer Verlag, Berlin, 2006, http://www.statslab. cam.ac.uk/ grg/books/rcm.html.

[23] _ Probability on Graphs, 2nd ed., Cambridge University Press, Cambridge, 2018, http://www.statslab.cam.ac.uk/ grg/books/pgs.html.

[24] G. R. Grimmett and D. R. Stirzaker, Probability and Random Processes, 3rd ed., Oxford University Press, Oxford, 2001.

[25] G. Kalai and S. Safra, Threshold phenomena and influence, Computational Complexity and Statistical Physics (A. G. Percus, G. Istrate, and C. Moore, eds.), Oxford University Press, New York, 2006, pp. 25-60.

[26] W. Kirsch, On Penrose's square-root law and beyond, Homo Oeconom. 24 (2007), 357-380.

[27] _ A mathematical view on voting and power, Mathematics and Society, Europ. Math. Soc., 2016, pp. 251-279.

[28] _ Effectiveness, decisiveness and success in weighted voting systems, (2017), https:// arxiv.org/abs/1706.08382. 
[29] W. Kirsch and J. Langner, The fate of the square root law for correlated voting, Voting Powers and Procedures (R. Fara, D. Leech, and M. Salles, eds.), Springer Verlag, Cham, 2014, pp. 147158.

[30] W. Kirsch, W. Słomczyński, and K. Życzkowski, Getting the votes right, European Voice (3-9 May 2007), http://chaos.if.uj .edu.pl/ karol/pdf/ZSZ07.pdf.

[31] Y. Koriyama, J.-F. Laslier, A. Macé, and R. Treibich, Optimal apportionment, J. Polit. Econ. 121 (2013), 584-608.

[32] M. Koster, S. Kurz, I. Lindner, and S. Napel, The prediction value, Social Choice and Welfare 48 (2017), 433-460.

[33] S. Kurz, On the inverse power index problem, Optimization 61 (2012), 989-1011.

[34] S. Kurz, N. Maaser, and S. Napel, On the democratic weight of nations, J. Polit. Econ. 125 (2017), 1599-1634.

[35] S. Kurz and S. Napel, Heuristic and exact solutions to the inverse power index problem for small voting bodies, Ann. Operat. Res. 215 (2014), 137-163.

[36] A. Laruelle and F. Valenciano, Voting and Collective Decision-Making, Cambridge University Press, Cambridge, 2008.

[37] I. Lindner and M. Machover, L. S. Penrose's limit theorem: proof of some special cases, Math. Soc. Sci. 47 (2004), 37-49.

[38] M. Machover, Penrose's square-root rule and the EU Council of Ministers: Significance of the quota, (2007), http://eprints.1se.ac.uk/2857/.

[39] S. Napel, Voting power, The Oxford Handbook of Public Choice, vol. 1, chap. 6, Oxford University Press, Oxford, 2019.

[40] L. Onsager, Crystal statistics. I. A two-dimensional model with an order-disorder transition, Phys. Rev. (2) 65 (1944), 117-149.

[41] L. Penrose, The elementary statistics of majority voting, J. Roy. Statist. Soc. 109 (1946), $53-57$.

[42] V. V. Petrov, Sums of Independent Random Variables, Springer Verlag, New York, 1975.

[43] F Pukelsheim, Proportional Representation, Springer Verlag, Cham, 2017.

[44] F. Pukelsheim and G. R. Grimmett, Degressive representation of Member States in the European Parliament 2019-2024, Representation 54 (2018), 147-158.

[45] F. Pukelsheim, G. R. Grimmett, V. Ramírez Gonzáles, W. Słomczyński, and K. Życzkowski, The Composition of the European Parliament, Policy Department for Citizens' Rights and Constitutional Affairs, Brussels, February 2017, PE 583.117, http://www.statslab.cam.ac. uk/ grg/papers/Composition2017-published.pdf.

[46] E. Ratzer, On the "Jagiellonian compromise" - voting in the European Union, (2006), http://www.inference.org.uk/ear23/voting/voting.pdf.

[47] L. Russo, On the critical percolation probabilities, Z. Wahrsch'theorie Verw. Gebiete 56 (1981), 229-237.

[48] I. G. Shevtsova, An improvement of convergence rate estimates in the Lyapunov theorem, Sov. Math. Dokl. 435 (2010), 862-864.

[49] W. Słomczyński and K. Życzkowski, Voting in the European Union: The square-root system of Penrose and a critical point, (2004), https://arxiv.org/abs/cond-mat/0405396.

[50] _ Penrose voting system and optimal quota, Acta Phys. Polonica 37 (2006), 3133-3143.

[51] . From a toy model to the double square root voting system, Homo Oeconom. 24 (2007), 381-399. 
[52] _ Jagiellonian Compromise — An alternative voting system for the Council of the European Union, Institutional Design vs Voting Power in the European Union (M. A. Cichocki and K. Życzkowski, eds.), Ashford Publishing, Abingdon, UK, 2010, pp. 43-57.

[53] A. Tomski, A toy model of a weighted voting game including two strong players, Mathematica Applicanda 42 (2014), 259-272.

[54] M. Widgrén, Enlargements and the principles of designing EU decision-making procedures, CESifo working paper series (2003), no. 903, https://papers.ssrn.com/sol3/papers.cfm? abstract_id=393863.

[55] K. Życzkowski and W. Słomczyński, Square root voting system, optimal threshold and $\pi$, Voting Powers and Procedures (R. Fara, D. Leech, and M. Salles, eds.), Springer Verlag, Cham, 2014, https: //arxiv.org/abs/1104.5213, pp. 127-146.

[56] K. Życzkowski, W. Słomczyński, and T. Zastawniak, Physicists for fairer voting, Physics World 19 (2006), 35-37, http://chaos.if.uj.edu.pl/ karol/pdf/ZSZ06.pdf.

Statistical Laboratory, Centre for Mathematical Sciences, Cambridge University, Wilberforce Road, Cambridge CB3 0WB, UK

School of Mathematics \& Statistics, The University of Melbourne, Parkville, VIC 3010, Australia

E-mail address: g.r.grimmett@statslab.cam.ac.uk

URL: http://www.statslab.cam.ac.uk/ grg/ 